Research Article

\title{
PER2 Regulates Reactive Oxygen Species Production in the Circadian Susceptibility to Ischemia/Reperfusion Injury in the Heart
}

\author{
Yaqian Weng, Hui Li, Lin Gao, Wenjing Guo, Shiyuan Xu $\mathbb{D}$, and Le Li $\mathbb{D}$ \\ Department of Anesthesiology, Zhujiang Hospital, Southern Medical University, Guangzhou, Guangdong 510282, China \\ Correspondence should be addressed to Shiyuan Xu; xsy998@smu.edu.cn and Le Li; lile11@126.com
}

Received 30 April 2021; Revised 15 August 2021; Accepted 24 August 2021; Published 8 October 2021

Academic Editor: Haobo Li

Copyright (c) 2021 Yaqian Weng et al. This is an open access article distributed under the Creative Commons Attribution License, which permits unrestricted use, distribution, and reproduction in any medium, provided the original work is properly cited.

\begin{abstract}
The main objective of this study was to investigate the diurnal differences in Period 2 (PER2) expression in myocardial ischemia-reperfusion (I/R) injury. We investigated diurnal variations in oxidative stress and energy metabolism after myocardial $\mathrm{I} / \mathrm{R}$ in vitro and in vivo. In addition, we also analyzed the effects of $\mathrm{H}_{2} \mathrm{O}_{2}$ treatment and serum shock in $\mathrm{H} 9 \mathrm{c} 2$ cells transfected with silencing RNA against Per2 (siRNA-Per2) in vitro. We used C57BL/6 male mice to construct a model of I/R injury at zeitgeber time (ZT) 2 and ZT14 by synchronizing the circadian rhythms. Our in vivo analysis demonstrated that there were diurnal differences in the severity of injury caused by myocardial infarctions, with more injury occurring in the daytime. PER2 was significantly reduced in heart tissue in the daytime and was higher at night. Our results also showed that more severe injury of mitochondrial function in daytime produced more reactive oxygen species (ROS) and less ATP, which increased myocardial injury. In vitro, our findings presented a similar trend showing that apoptosis of H9c2 cells was increased when PER2 expression was lower. Meanwhile, downregulation of PER2 disrupted the oxidative balance by increasing ROS and mitochondrial injury. The result was a reduction in ATP and failure to provide sufficient energy protection for cardiomyocytes.
\end{abstract}

\section{Introduction}

Acute myocardial infarction (AMI) is a major cause of morbidity and mortality worldwide. Emergency treatment for AMI has been revolutionized by timely reperfusion therapy [1]. Reperfusion is "a double-edged sword." It not only provides oxygen and nutrients for ATP production and washes out toxic metabolite accumulation but also induces a burst of reactive oxygen species (ROS) leading to a second injury or dominant injury $[2,3]$. However, there is no therapy directly targeting the injury caused by reperfusion.

The frequency of the onset of AMI is not random and has been reported to have a circadian variation, with the peak in the early morning [4]. Also, circadian rhythmicity of the heart has been reported in multiple animal studies $[5,6]$. In particular, recent studies using mutant mouse models with cardiomyocyte-specific defects in the core clock machinery suggested that susceptibility of the myocardium itself varies in a time-of-day-specific fashion [7]. However, the molecular mechanism remains poorly understood.

The circadian clock within the heart modulates myocardial metabolism, which in turn facilitates anticipation of diurnal variations in workload, stimuli, and/or the energy supply-todemand ratio [8]. ROS, as an intrinsic by-product of oxidative phosphorylation, also undergoes circadian rhythms [9]. It may be beneficial for the organism to coordinate catabolism/energy utilization and ROS clearance in a circadian fashion. Nonetheless, a master transcriptional regulator of both processes is not known.

Period 2 (PER2), a molecular component of the mammalian circadian clock, plays a key role in controlling the circadian rhythms in physiology and behavior [10]. Recently, increasing evidence has suggested that PER2 mediates the cardiac protection effect $[11,12]$. Per $^{-/-}$mice have larger infarct sizes with deficient lactate production during myocardial ischemia [13], suggesting that PER2 protects 
the heart by regulating catabolism/energy. PER2 was also reported to be involved in regulating the cellular response to oxidative stress in mouse embryonic fibroblasts [14], pancreatic islets [15], and other organs. In the present study, we discovered that the circadian rhythm protein PER2 mediates susceptibility to I/R insult in a time-of-day-dependent fashion by regulating energy utilization and ROS clearance.

\section{Methods}

2.1. Animals. The use of specific pathogen-free C57BL/6J mice was approved by the Institutional Animal Care Committee at Zhujiang Hospital of Southern Medical University, China. Mice were housed in a temperature- and humiditycontrolled specific pathogen-free facility with a $12 \mathrm{~h}$ light/dark cycle and ad libitum access to water and standard laboratory rodent chow.

2.2. Ischemia-Reperfusion (I/R) Surgery. I/R studies were performed on 10-12-week-old male mice. Mice were anesthetized with sodium pentobarbital ( $70 \mathrm{mg} / \mathrm{kg}$ intraperitoneally), placed on a temperature-controlled heating pad, and ventilated through endotracheal intubation. A standard 3-lead EKG and a rectal temperature probe were placed for monitoring. A thoracotomy was performed, and the heart was exposed by stripping the pericardium. The left anterior descending coronary artery was encircled by an 8-0 Prolene suture, and ischemia was induced by tightening the suture, which was confirmed visually by blanching of the distal cardiac tissue and ST-elevation on the electrocardiogram [16]. The suture was released after $30 \mathrm{~min}$ to allow reperfusion. The mice were maintained on ventilation until recovery from anesthesia. Infarct size was assessed $24 \mathrm{~h}$ postreperfusion using $1 \%$ Evans blue and 2\% triphenyltetrazolium chloride (TTC) staining [17] and was expressed as a percentage of the at-risk area.

2.3. Echocardiographic Assessment. At the end of reperfusion, mice were reanesthetized with isoflurane, fixed on the experimental table, and studied with an echocardiography system (Vevo 2100, a high-resolution ultrasound echocolor Doppler system from VisualSonics, Canada). The following variables were measured and averaged during three consecutive cardiac cycles: left ventricular end-systolic diameter (LVESD), left ventricular end-diastolic diameter (LVEDD), left ventricular end-systolic volume (LVSV), and left ventricular end-diastolic volume (LVDV). The left ventricular ejection fraction (LVEF) and left ventricular fractional shortening (LVFS) values were converted by the Simpson method with the following formula: $\mathrm{LVEF}=(\mathrm{LVDV}-\mathrm{LVSV}) / \mathrm{LVDV} \times$ $100 \% ;$ LVFS $=($ LVDD - LVSD $) / L V D D \times 100 \% . \quad$ LVEF and LVFS were used as parameters indicating cardiac function [18]. The experiment was conducted three times, and the mean value was obtained.

2.4. Cell Culture and Serum Shock-Induced Circadian Rhythms. H9c2 cells were purchased from the National Collection of Authenticated Cell Culture (China). H9c2 cells were grown in an incubator in a DMEM-F12 medium (Corning) complemented with $10 \%$ fetal bovine serum (FBS; GIBCO) at $37^{\circ} \mathrm{C}$ in $5 \% \mathrm{CO}_{2}$. Cells were subcultured every 4 days prior to the experiment in 6-well plates; they were confluent after 4 days. The cells were treated with $50 \%$ horse serum (50\% DMEM-F12 and 50\% horse serum, BI) for $2 \mathrm{~h}$ and were then washed twice with tepid DMEM-F12 without serum. Samples were taken every $4 \mathrm{~h}$ for $24 \mathrm{~h}$ following serum shock. Harvested cells were immediately frozen at $-80^{\circ} \mathrm{C}$ until RNA isolation or protein extraction [19, 20].

2.5. $\mathrm{H}_{2} \mathrm{O}_{2}$ Treatment after Serum Shock in Cells Transfected with siRNA-Per2. For transfection experiments, $1 \times 10^{5}$ H9c2 cells were plated in 6-well plates the day before transfection. Transfections were performed using $1 \mu \mathrm{g}$ siRNA oligo targeting Per2 and $5 \mu \mathrm{l}$ Lipofectamine 3000 (Thermo Fisher, USA) in serum-free media per well, according to the manufacturer's protocol. After $12 \mathrm{~h}$ of transfection, the cells were treated with $50 \%$ horse serum for $2 \mathrm{~h}$. At the end point or $12 \mathrm{~h}$ after serum shock, cells were treated with $\mathrm{H}_{2} \mathrm{O}_{2}$ for $2 \mathrm{~h}[21,22]$.

2.6. TUNEL Staining. Cardiomyocyte apoptosis was assessed in heart sections by terminal deoxynucleotidyl transferase dUTP nick end labeling (TUNEL). The TUNEL mix (KeyGEN Biotech) contained $50 \mu \mathrm{l}$ enzyme solution and $450 \mu \mathrm{l}$ labeling solution. Heart sections were incubated with $50 \mu \mathrm{l}$ TUNEL mix at $37^{\circ} \mathrm{C}$ for $1 \mathrm{~h}$. The sections were then washed twice with PBS and stained with DAPI. After washing with PBS for another three times, the sections were observed by fluorescence microscopy with $\operatorname{Ex}(\lambda) 450-500 \mathrm{~nm}, \operatorname{Em}(\lambda)$ $515-565 \mathrm{~nm}$ for TUNEL and $\operatorname{Ex}(\lambda) 359 \mathrm{~nm}, \operatorname{Em}(\lambda) 461 \mathrm{~nm}$ for DAPI (NIKON T12-E, Japan). The apoptosis ratio was calculated as apoptotic cell number (green)/total cell number (blue) $\times 100 \%$.

2.7. Determination of ROS Production. Dihydroethidium (DHE, BestBio, China) staining was used to detect ROS levels in heart tissue. Fresh mouse heart tissue samples were embedded in an OCT compound (Thermo Fisher). Cryopreserved sections were then loaded with $500 \mu \mathrm{M}$ DHE following the manufacturer's instructions. Oxidized DHE was excited at $543 \mathrm{~nm}$, and emission was collected with a LP $560 \mathrm{~nm}$ filter using the NIKON T12-E fluorescence microscope.

Intracellular ROS levels in $\mathrm{H} 9 \mathrm{c} 2$ cells were determined by measuring the oxidative conversion of cell-permeable $2^{\prime}, 7^{\prime}$-dichlorofluorescein diacetate (DCFH-DA) to fluorescent dichlorofluorescein (DCF). The cells were washed with D-Hank's balanced salt solution (HBSS) and incubated with $\mathrm{DCFH}-\mathrm{DA}$ at $37^{\circ} \mathrm{C}$ for $20 \mathrm{~min}$. Then, fluorescence was detected by flow cytometry at $\operatorname{Ex}(\lambda) 488 \mathrm{~nm}, \operatorname{Em}(\lambda)$ $525 \mathrm{~nm}$ (Beckman Coulter), which would collect and analyze ten thousand cells in each flow cytometric assay.

2.8. Determination of ATP Production. Adenosine $5^{\prime}$-triphosphate (ATP; Nanjing Jiancheng Bioengineering Institute, China) staining was used to detect ATP levels in heart tissue and H9c2 cells according to the manufacturer's instructions, using a microplate reader (Thermo Fisher).

2.9. Mitochondrial Membrane Potential (MMP) Measurement. The MMP of H9c2 cells was measured using the fluorescent probe, JC-1 (KeyGEN Biotech). The cells were rinsed with 
HBSS and incubated with JC-1 $(10 \mu \mathrm{M})$ at $37^{\circ} \mathrm{C}$ for $30 \mathrm{~min}$. Afterwards, the cells were rinsed with HBSS once again. Fluorescent intensity of the JC- 1 monomers and aggregates was detected by flow cytometry with $\operatorname{Ex}(\lambda) 490 \mathrm{~nm}, \operatorname{Em}(\lambda)$ $530 \mathrm{~nm}$ for monomers and $\operatorname{Ex}(\lambda) 525 \mathrm{~nm}, \operatorname{Em}(\lambda) 590 \mathrm{~nm}$ for aggregates (Beckman Coulter), and ten thousand cells were collected and analyzed each time.

2.10. Quantitative RT-PCR. Heart samples or H9c2 cells were disrupted/homogenized in a TRIzol reagent (Accurate Biotechnology, China) with a Tissue-lyzer (LUKYM, China), and total RNA was extracted according to the manufacturer's directions. RNA was reverse transcribed using the Accurate Biotechnology reverse transcription kit following the manufacturer's instructions. Quantitative PCR was performed using the SYBR Green Premix Pro Taq HS qPCR Kit (Accurate Biotechnology) using a two-step PCR amplification standard procedure on a Bio-Rad (USA) CFX connect system. Relative expression was calculated using the $\Delta \Delta \mathrm{Ct}$ method with normalization to Gapdh. Specific primer/probe sequences are shown below. Primer sequences are listed in Table 1 of supplementary data.

2.11. Immunoblots and Antibodies. Whole-cell lysates or isolated mitochondrial lysates were prepared by homogenizing the basal regions of the hearts in RIPA buffer (Invitrogen, USA) supplemented with protease 13 inhibitors (Invitrogen). Immunoblots were prepared using the antibodies listed below and were normalized to GAPDH (Sigma, USA). Antibodies used for western blots are listed in Table 2 of supplementary data.

2.12. Statistics. The results are presented as the means \pm SEM . Two-tailed Student's $t$-tests were used to compare the difference between two groups. One-way or two-way ANOVA with Bonferroni correction was used for multiple comparisons. Statistical significance was defined as $P<0.05$.

\section{Results}

3.1. Diurnal Differences in Myocardial Infarction Injury. The infarct area of cardiac tissue in each group was detected by TTC and Evans blue staining and quantified using ImageJ. The results showed that the area at risk in the left ventricle (AAR/LV) after I/R was not significantly different among the four groups (Figure 1(b), left). However, the infarct area in the area at risk (IR/AAR) after I/R at ZT2 was larger than at ZT14, while no difference was observed at ZT8 or ZT20 (Figure 1(b), right). As shown in Figure 1(c), ischemia followed by reperfusion induced significant myocardial injury as denoted by the white infarct size in mice. In contrast, ZT14 significantly decreased the ratio of infarct area/risk area (MI/AAR) compared with the ZT2 group (Figure 1(c), right).

M-mode ultrasound was used to evaluate changes in cardiac function of 10 12-week-old mice in each group, $24 \mathrm{~h}$ after myocardial I/R. Typical M-mode ultrasounds showed that compared with the sham group without I/R at zeitgeber time (ZT) 2 or ZT14, the ejection fraction (EF) of the left ventricle was decreased in mice $24 \mathrm{~h}$ after I/R. In addition, the EF in mice after I/R at ZT2 was lower than that in mice after I/R at ZT14 Figure 1(d), left). At the same time, fraction shortening (FS) of the left ventricle in mice after I/R at ZT2 was lower than that in mice with I/R at ZT14 (Figure 1(d), right), which showed that mice receiving I/R at ZT2 had worse cardiac systolic function than at ZT14.

3.2. Diurnal Differences in Oxidative Stress and Energy Metabolism. Histological analysis and immunofluorescence staining were performed to further evaluate myocardial cell activity. Typical results of TUNEL staining showed that cell apoptosis of myocardial tissue after I/R at ZT2 was more extensive than that observed in the ZT14 group (Figure 2(a)). These results suggested that I/R at ZT2 could significantly increase myocardial cell apoptosis (Figure 2(b)).

DHE staining results showed that ROS was increased in myocardial cells after I/R, where the ROS level in the ZT2 group was higher than that in the ZT14 group (Figure 2(c)).

ATP determination assays performed in each group showed that without I/R, sham-ZT14 mice produced more ATP than sham-ZT2 mice, although the difference was not statistically significant. In contrast, $24 \mathrm{~h}$ after I/R at ZT2, ATP content was lower than in sham-ZT2 mice. The ATP content $24 \mathrm{~h}$ after I/R at ZT14 was higher than that in the sham-ZT14 group, which indicated that mice produced more ATP at ZT14 while producing less ATP at ZT2 (Figure 2(d)).

3.3. Diurnal Differences Affected the Expression of PER2 and CPT1A. mRNA and protein were collected from the mice every $4 \mathrm{~h}$ for $24 \mathrm{~h}$. The transcription level of Per 2 showed a distinct rhythmic pattern for nearly $24 \mathrm{~h}$ (Figure $3(\mathrm{a})$ ). The transcription level of Per2 increased from ZT2, reaching a peak value near ZT14, then decreased again to a low value near ZT2 (Figure 3(a)). The change in PER2 protein levels was consistent with the mRNA results. Mice displayed a rhythm in PER2 protein expression with peak values at ZT14 and lowest values at ZT2 as shown by western blotting (Figure 3(b)). The protein expression of PER2, carnitine palmitoyltransferase 1A (CPT1A), and pyruvate dehydrogenase PDH-E1B (PDHB) after I/R was also detected by western blotting (Figure 3(c)). The results showed that the protein expression of PER2 and CPT1A was similar under the same treatment conditions and decreased after I/R at ZT2, while increasing after I/R at ZT14. PDHB showed no difference after I/R.

3.4. Downregulation of PER2 Increased Injury. H9c2 cells that were transfected with siRNA-Per2 were collected after $\mathrm{H}_{2} \mathrm{O}_{2}$ treatment and serum shock at $0 \mathrm{~h}$ and $12 \mathrm{~h}$. mRNA was collected every $4 \mathrm{~h}$ for $24 \mathrm{~h}$. The transcription level of Per2 showed a distinct rhythmic pattern for nearly $24 \mathrm{~h}$ (Figure 4(a)). From $0 \mathrm{~h}$ after serum shock, the transcription level of Per 2 decreased until reaching a low value near $12 \mathrm{~h}$ (Figure 4(a)). After transfection with siRNA-Per2, there was a significant reduction in PER2, shown in Figure 4(b).

ATP determination assays were also performed for each group. After $\mathrm{H}_{2} \mathrm{O}_{2}$ treatment, ATP was decreased at $12 \mathrm{~h}$ but was increased at $0 \mathrm{~h}$ (Figure $4(\mathrm{c})$ ). ATP levels were 


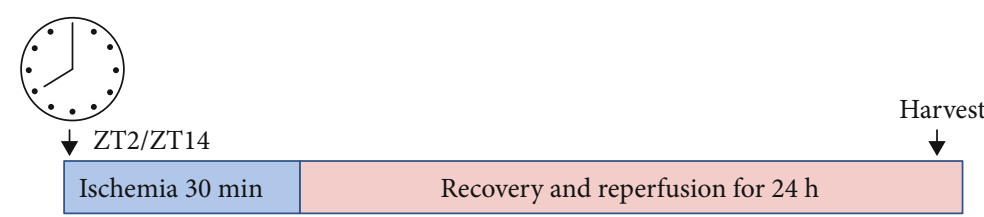

(a)
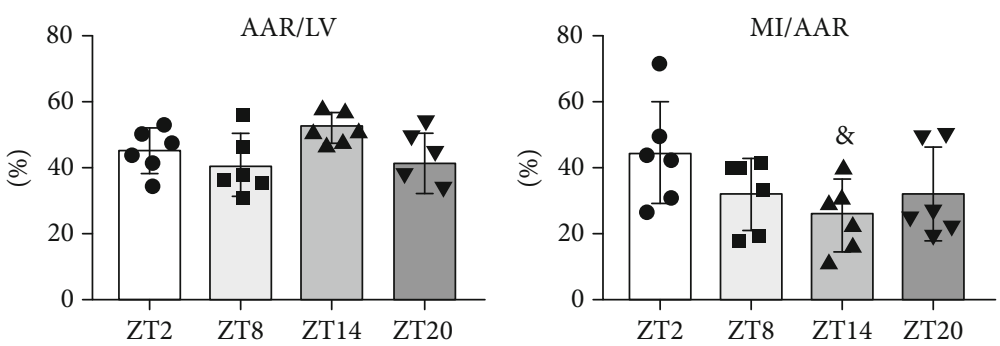

(b)

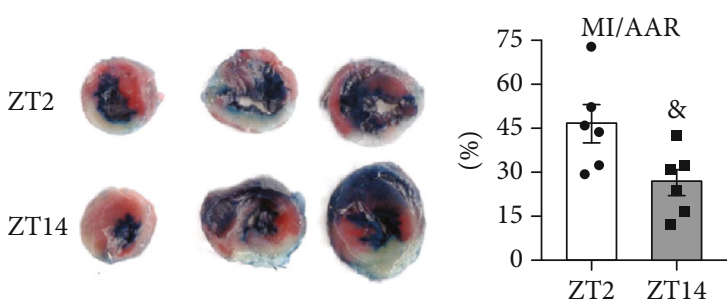

(c)

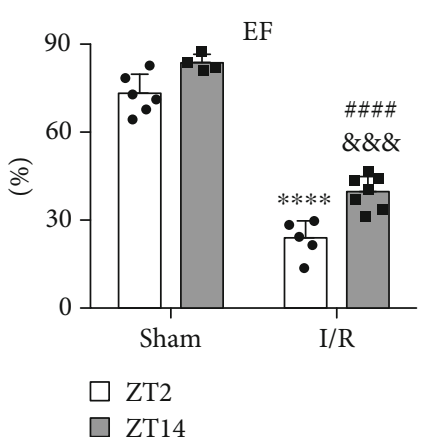

(d)

Figure 1: Circadian differences in susceptibility of the heart to I/R injury. (a) Experimental design. Mice were subjected to a protocol of $30 \mathrm{~min}$ of ischemia followed by $24 \mathrm{~h}$ of reperfusion starting at two time points, ZT2 or ZT14. ZT: zeitgeber time; ZT0: lights on. (b) Percent of area at risk over area of the left ventricle (middle). Percent of infarct over area at risk (right). AAR: area at risk; LV: left ventricle; MI: myocardial infarction $\left(n=6,{ }^{8} P<0.05\right.$ vs. ZT2). (c) Infarct size. Representative Evans blue and TTC staining (left). Percent of infarct over area at risk (right). White: infarct; red: viable; white+red: area at risk; blue: retrograde Evans blue staining, area not at risk ( $n=6,{ }^{\&} P<0.05$ vs. IR-ZT2, two-tailed Student's $t$-test). (d) Cardiac systolic function of mice with I/R at ZT2 and ZT14. EF of the left ventricle (left), FS of the left ventricle (right). EF: ejection fraction; FS: fraction shortening; sham: sham surgery; I/R: ischemia-reperfusion. ( $n=4-7,{ }^{* * *} P<0.0001$ and ${ }^{* * *} P<0.001$ vs. sham-ZT2; ${ }^{\# \# \#} P<0.0001$ vs. sham-ZT14; ${ }^{\&} P<0.05$ and ${ }^{\& \& \&} P<0.001$ vs. IR-ZT2, two-tailed Student's $t$-tests). Alpha was set as 0.05 . Data are presented as the mean \pm SEM. The Holm-Sidak method was used to correct for multiple $t$-tests.

significantly decreased after siRNA-Per2 transfection (Figure 4(c)), suggesting that downregulation of PER2 decreased ATP.

Histological analysis and immunofluorescence staining were performed to further evaluate H9c2 cell activity. The results of TUNEL staining showed that cellular apoptosis of $\mathrm{H} 9 \mathrm{c} 2$ cells after $\mathrm{H}_{2} \mathrm{O}_{2}$ treatment at $0 \mathrm{~h}$ was less than apoptosis at $12 \mathrm{~h}$ after serum shock (Figure $4(\mathrm{~d})$ ). After siRNAPer2 transfection, cell death increased compared with the control group, but there was no significant difference at $0 \mathrm{~h}$ or $12 \mathrm{~h}$ after serum shock whether the cells were treated with $\mathrm{H}_{2} \mathrm{O}_{2}$ or not. These results indicated that in $\mathrm{H} 9 \mathrm{c} 2$ cells, $12 \mathrm{~h}$ of serum shock and siRNA-Per2 transfection could both increase myocardial cell apoptosis (Figure 4(d)).

ROS detected by flow cytometry showed that in cells treated with $\mathrm{H}_{2} \mathrm{O}_{2}$, ROS increased significantly (Figure 4(e)). At the same time, ROS increased after siRNA-Per2 transfection, whether or not the cells were treated with $\mathrm{H}_{2} \mathrm{O}_{2}$ (Figure 4(e)).
Flow cytometric analysis was performed to identify quantitatively the MMP in H9c2 cells, which is one of the important hallmarks of mitochondrial damage. The contour plots in Figure 4(f) showed that the fluorescence for high MMP in Q2 was shifted to low MMP in Q3. $\mathrm{H}_{2} \mathrm{O}_{2}$ treatment showed a strong ability to dissipate the MMP, and cells after $12 \mathrm{~h}$ of serum shock showed more dissipation of MMP than cells at $0 \mathrm{~h}$ (Figure 4(f)). Furthermore, transfection of siRNA-Per2 resulted in more dissipation of MMP. These results suggested that the mitochondrial membrane was impaired by $\mathrm{H}_{2} \mathrm{O}_{2}$, siRNA-Per 2 transfection, and $12 \mathrm{~h}$ of serum shock (Figure 4(e), lower panel, right figure).

mRNA expression of Per2, Cpt1a, and Pdhb in H9c2 cells was quantified by RT-PCR analysis (Figure $4(\mathrm{~g})$ ). The results showed that the expression of Per2 and Cpt1a was similar under the same treatment conditions. Expression of these genes was higher before serum shock compared with $12 \mathrm{~h}$ later and decreased after $\mathrm{H}_{2} \mathrm{O}_{2}$ treatment or siRNAPer2 transfection. 

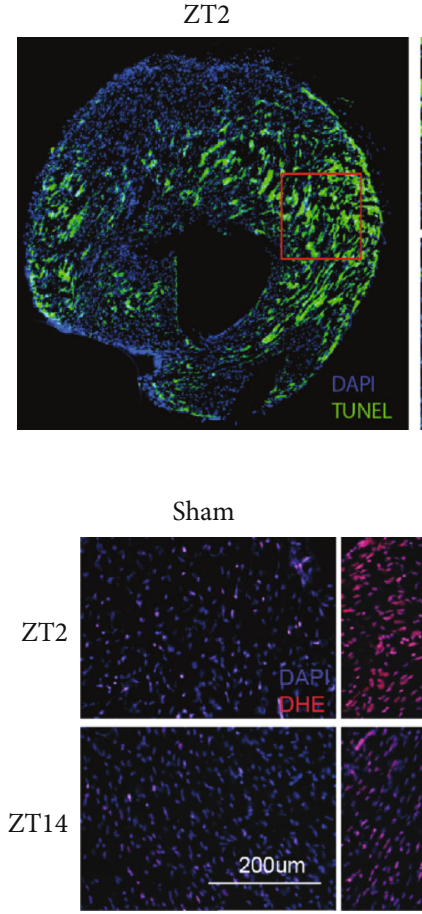

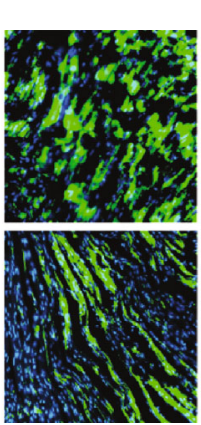

(a)

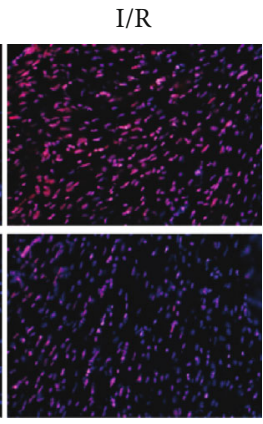

(c)
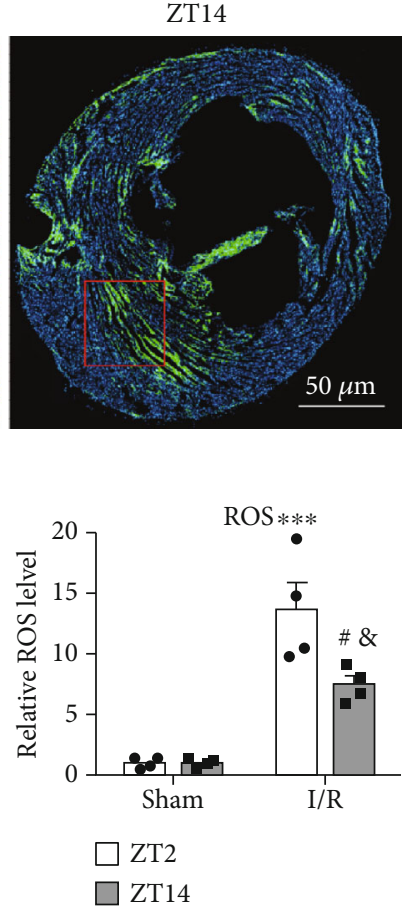

ZT14

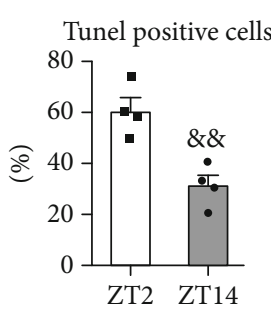

(b)

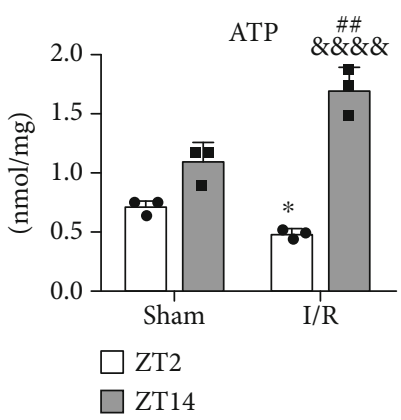

(d)

Figure 2: Circadian differences in oxidative stress and energy metabolism. (a) Apoptotic cells were determined by TUNEL staining. Representative apoptotic cells of mouse hearts after I/R injury at ZT2 (left) and ZT14 (right). Magnified area of interest from mouse heart after I/R injury at ZT2 (up middle) and ZT14 (bottom middle). (b) Quantitative analysis of TUNEL cells ( $n=4$, ${ }^{\& \&} P<0.01$ vs. IR-ZT2, two-tailed Student's $t$-test). (c) ROS in the hearts of mice. Representative DHE staining of the peri-infarct area of mouse hearts after $24 \mathrm{~h}$ IR at ZT2 and ZT14 (left), quantitative analysis of ROS (middle) $\left(n=4,{ }^{* * *} P<0.001\right.$ vs. sham-ZT2; ${ }^{*} P<0.05$ vs. sham-ZT14; ${ }^{8} P<0.05$ vs. IR-ZT2, two-tailed Student's $t$-tests). (d) ATP change in mouse hearts with I/R injury $\left(n=3,{ }^{*} P<0.05\right.$ vs. sham-ZT2; ${ }^{\# \#} P<0.01$ vs. sham-ZT14; ${ }^{\& \& \& \&} P<0.0001$ vs. IR-ZT2, two-tailed Student's $t$-tests). Alpha was set as 0.05 . Data are presented as the means \pm SEM. The Holm-Sidak method was used to correct for multiple $t$-tests.

\section{Discussion}

Taken as a whole, the current findings suggested that PER2 was the basis of circadian changes in cardiac tolerance to I/R and that knockdown of PER2 expression could increase I/R damage. Furthermore, PER2 likely acted downstream as a mediator underlying the transcriptional clock mechanism in cardiac susceptibility to $\mathrm{I} / \mathrm{R}$ damage by regulating CPT1A as a key enzyme in fatty acid metabolism and oxidative stress levels. This result is important because it focuses on energy metabolism and reactive oxygen species as potential therapeutic targets, which represent physiological oscillations and metabolic changes in the cardiovascular system itself as the basis for cardiac protection.

Many studies have implicated circadian rhythm in the brain [23], liver [24], lung [25], kidney [26], and heart [27]. Epidemiologic studies have reported circadian rhythmicity in the incidence and the size of myocardial infarctions which were higher during the day and lower at night [28]. In addition to external factors such as neurohormonal regulation, platelet aggregation, and vascular activities, the vulnerability of the myocardium shows a diurnal variation, but the molecular mechanism is not clear.

In this study, we found that mice showed a bigger infarct at ZT2 (Figure 1) and were susceptible to cardiac I/R damage at the end of the light phase. This result was similar to a previous basic research report of Li et al. [7], who suggested that a deficiency of the oscillating transcription factor KLF15 may specifically cause NAD+ deficiency during the sleepto-active transition and may increase the susceptibility of the heart muscle to I/R injury. Interestingly, other studies have shown opposite results, with ZT14/ZT12 having larger infarcts than ZT2/ZT0 [29]. As Rotter et al. reported, cardiac damage from ischemia/reperfusion was greatest at the transition from sleep to activity, and Rcan 1 control of calcineurin activation was necessary for the daily oscillations of the heart against injury [30]. However, Rcan1 KO was equally more susceptible to damage from $\mathrm{I} / \mathrm{R}$ at the end of the light phase. We hypothesized that these differences in results might be related to the timing of ischemia and reperfusion, reflecting the fundamental difference in the cell injury mechanism between ischemia and reperfusion.

Combined with previous studies, diurnal variations in the onset of myocardial infarction are well established. The influence of the circadian clock cannot be ignored in heart I/R studies. Various proteins compose the circadian clock, including PER2, CRY, CLOCK, and BMAL [31]. PER2 can "buffer" or adjust the antagonism between CLOCK/BMAL1 and CRY, so that CLOCK reaches a self-limiting, rhythmic cycle [32]. Through RT-PCR and western blots, we saw that 


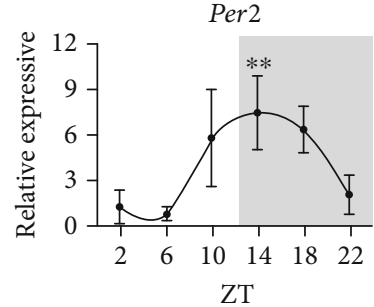

(a)

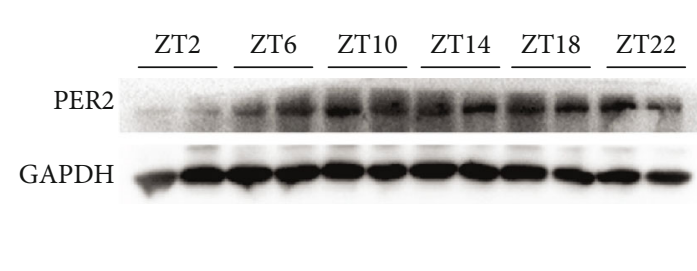

(b)

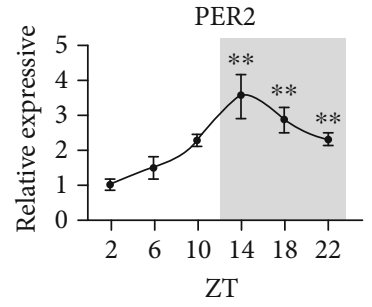

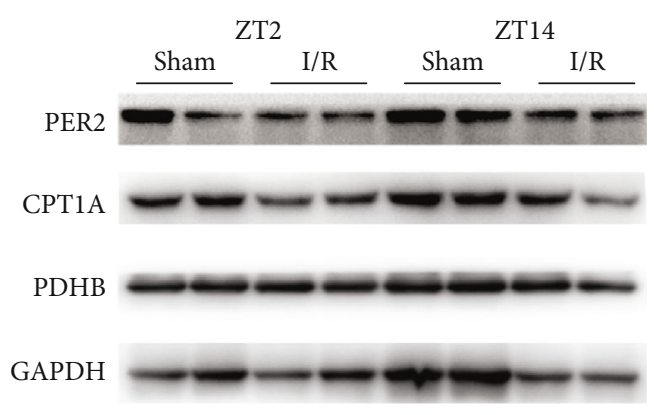

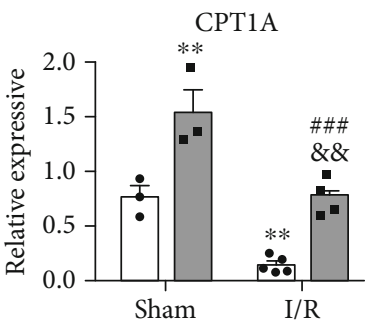

ZT2

ZT14
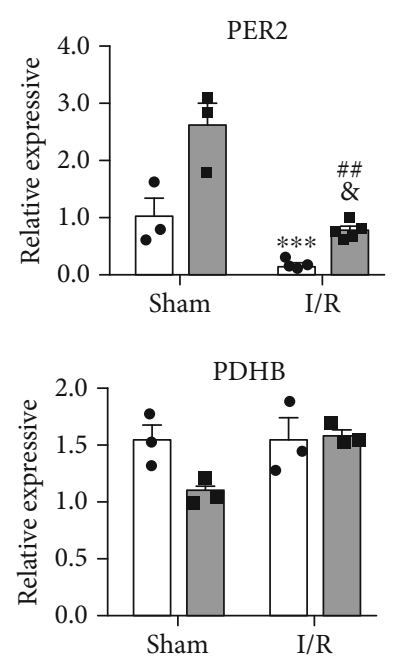

(c)

FIgure 3: PER2 in circadian I/R susceptibility of the heart. Per 2 mRNA (a) and (b) protein expression in mouse heart during a 24 h day. Quantification was normalized to GAPDH. ZT2 was set to $1\left(n=3\right.$ per time point, ${ }^{* *} P<0.01$ and ${ }^{* * *} P<0.001$ vs. ZT2; one-way ANOVA with Bonferroni correction). (c) PER2 and potential target levels were determined by immunoblots with or without I/R injury at ZT2 and ZT14. Quantification of PER2, CPT1, and PDHB was normalized to GAPDH $(n=3-5, * * P<0.01$ and $* * * P<0.001$ vs. sham-ZT2; ${ }^{\# \#} P<0.01$ and ${ }^{\# \# \#} P<0.001$ vs. sham-ZT14; ${ }^{\circledR} P<0.05$ and ${ }^{\& \&} P<0.01$ vs. I/R-ZT2, two-tailed Student's $t$-test). Alpha was set as 0.05 . Data are presented as the means \pm SEM. The Holm-Sidak method was used to correct for multiple $t$-tests.

in C57BL/6 mice, PER2 expression showed a distinct rhythmic pattern for nearly $24 \mathrm{~h}$ (Figure 3 ), and the valley of PER2 expression coincided with the time when the damage resistance of the heart was reduced after the transition from dark to light. Another study has shown that mice with Per2-/- had enhanced tissue damage from myocardial ischemia and lacked the capacity to enhance oxygen-efficient glycolysis [33].

Our results suggested that the absence of PER2 was associated with mitochondrial injury, which then led to less ATP and more ROS production, thus increasing cell injury. After cardiac ischemia-reperfusion, the oxidative and antioxidant capacities of myocardial cells are unbalanced, resulting in a state of "oxidative stress," which plays an important role in the injury caused by myocardial infarction. Reactive oxygen species and oxidative stress have been reported to play important roles in the progression after myocardial infarction [34]. Wu et al. demonstrated that acacetin stimulated AMPK, which mediates activation of the Nrf2 signaling pathway in cardiomyocyte protection against hypoxia/reox- ygenation injury by increasing the antioxidants heme oxygenase 1 (HO-1), SOD1, and SOD2, reducing ROS production and thereby effectively inhibiting hypoxia/reoxygenation injury [35]. As reported by Ortiz et al., the use of thyroid hormone (TH) as a therapeutic alternative revealed cardioprotective effects after AMI, including decreased oxidative stress, while carvedilol and $\mathrm{TH}$ coadministration improved redox balance and cardiac function after AMI [36]. Finding a promising drug which protects cardiomyocytes against hypox$\mathrm{ia} /$ reoxygenation may help manage ischemic cardiac disorders.

To pinpoint mitochondrial processes that may be under circadian gene PER2 control, we examined two central mitochondrial metabolic pathways of carbohydrate metabolism and fatty acid uptake. The rate-limiting step in mitochondrial carbohydrate metabolism is carried out by the PDC, a multiprotein complex that catalyzes the oxidative decarboxylation of pyruvate [37]. We found that several components of the PDC, namely, the catalytic pyruvate dehydrogenase PDHB, PDH-E2 (DLAT), and the regulatory subunit PDHX, 


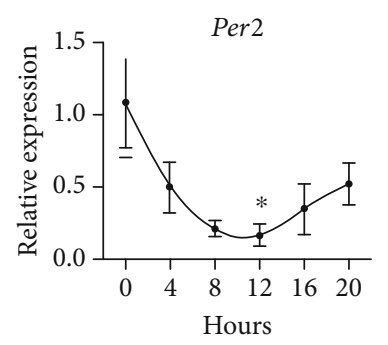

(a)

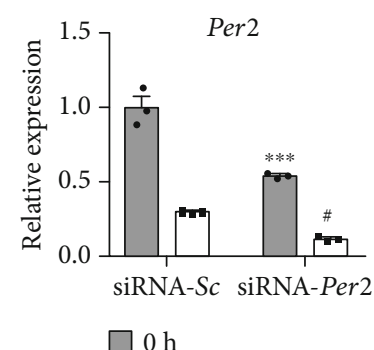

$\square 12 \mathrm{~h}$

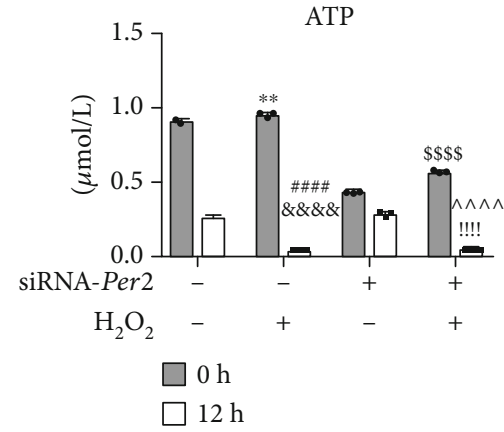

(c)

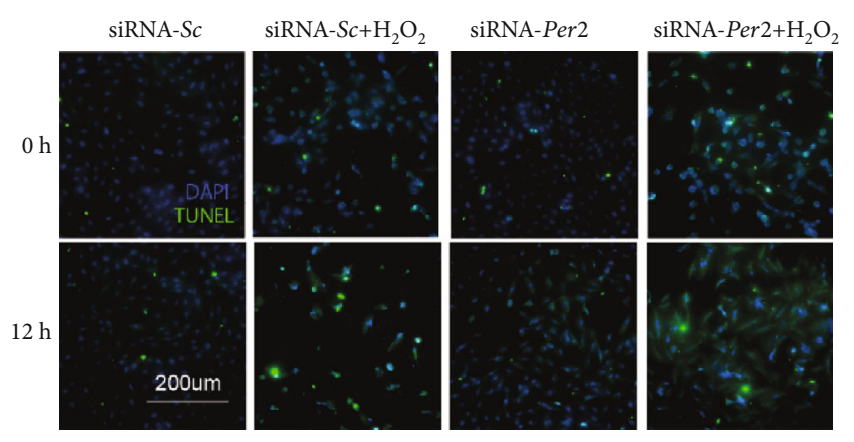

$\begin{aligned} & \square 0 \mathrm{~h} \\ & \square 12 \mathrm{~h}\end{aligned}$

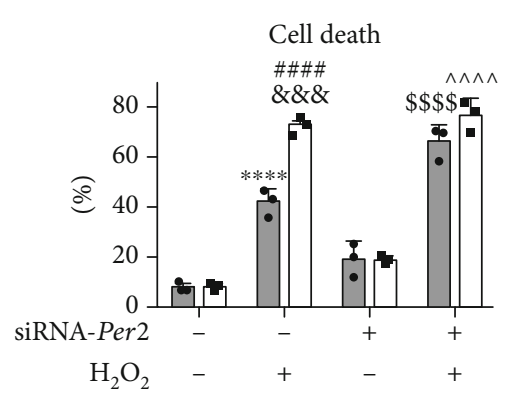

(d)

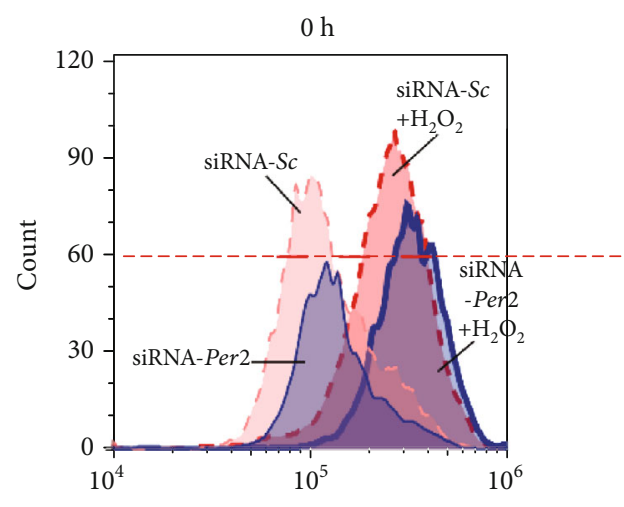

Fluorescence intensity

ROS
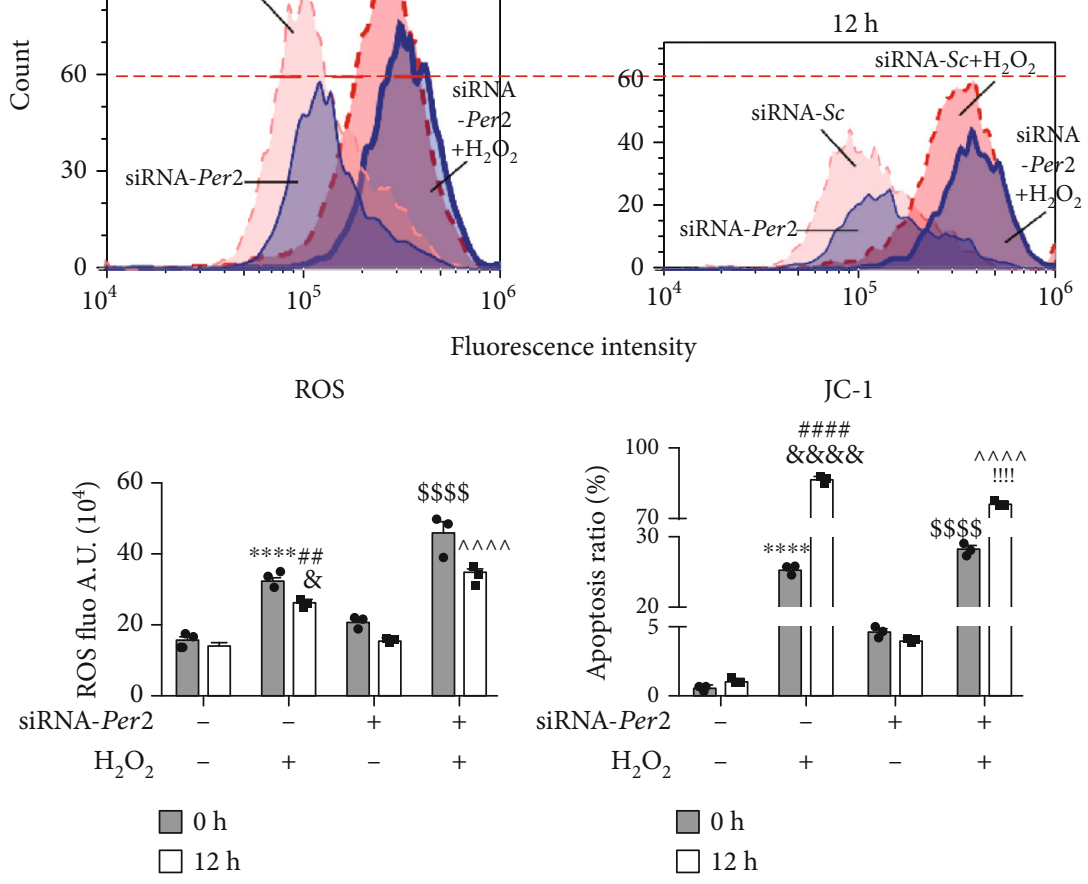

(e)

FIgURE 4: Continued. 


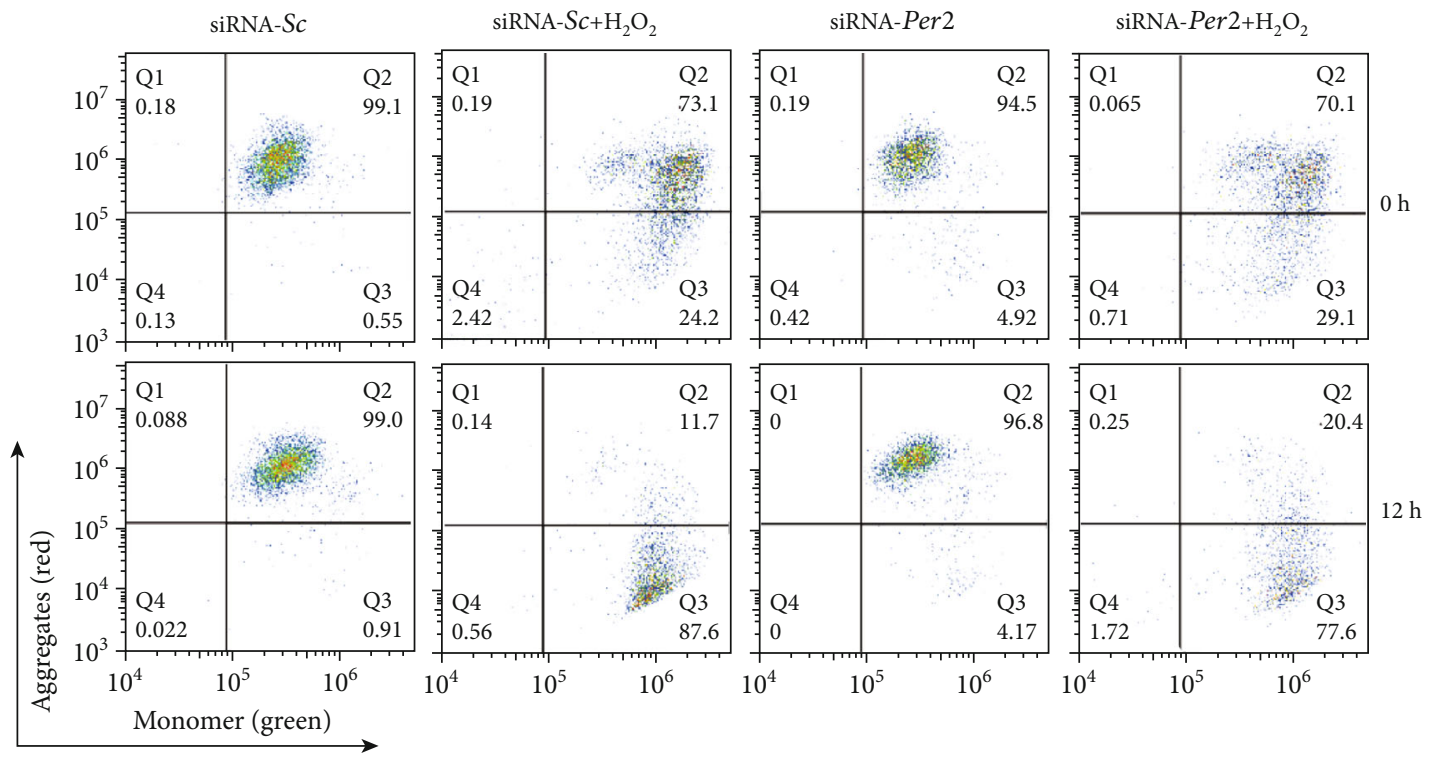

(f)
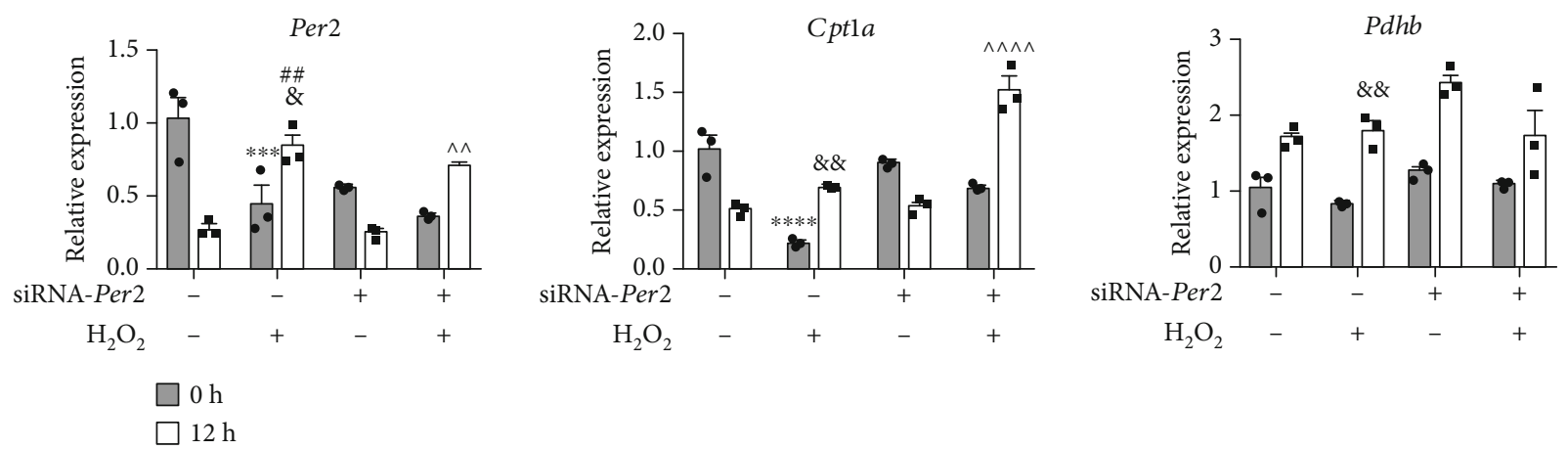

(g)

FIgURE 4: PER2 deficiency in the tolerance of $\mathrm{H} 9 \mathrm{c} 2$ cells to $\mathrm{H}_{2} \mathrm{O}_{2}$ treatment after serum shock. (a) Per2 mRNA expression during $24 \mathrm{~h}$ after serum shock ( $n=3$ per time point, ${ }^{*} P<0.05$ vs. $0 \mathrm{~h}$ after serum shock, one-way ANOVA with Bonferroni correction). (b) Per2 mRNA expression levels in H9c2 cells after knockdown of Per 2 using siRNA $\left(n=3,{ }^{* * *} P<0.001\right.$ vs. siRNA-Sc- 0 hr, ${ }^{\#} P<0.05$ vs. siRNA-Sc-12 hr). (c-g) H9c2 cells were transfected with siRNA-Sc or siRNA-Per $24 \mathrm{~h}$ before serum shock. Transfected cells were treated with $\mathrm{H}_{2} \mathrm{O}_{2}$ for $2 \mathrm{~h}$ at the ending point or $12 \mathrm{~h}$ after serum shock. (c) ATP change in transfected $\mathrm{H} 9 \mathrm{c} 2$ cells treated with $\mathrm{H}_{2} \mathrm{O}_{2}$ at $0 \mathrm{~h}$ and $12 \mathrm{~h}$ after serum shock. (d) Cell death determined by TUNEL staining. Representative image (left) and quantification (right). (e) ROS in cells. Flow cytometry images (upper), quantitative analysis of ROS (lower panel, left figure). (f) Flow cytometry of JC-1 in cells. Representative images (f), quantitative analysis of JC-1 ((e), lower panel, right figure). (g) Per2 and potential target levels in H9c2 cells were determined by RT-PCR at $0 \mathrm{~h}$ and $12 \mathrm{~h}$. Quantification of Per2, Cpt1a, and $P d h b$ was normalized to GAPDH $\left(n=3,{ }^{* *} P<0.01,{ }^{* * *} P<0.001\right.$, and ${ }^{* * * *} P<0.0001$ vs. cells transfected

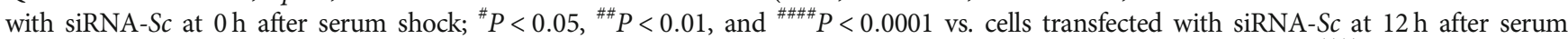
shock; ${ }^{\&} P<0.05$ and ${ }^{\& \& \& \&} P<0.0001$ vs. cells transfected with siRNA-Sc treated with $\mathrm{H}_{2} \mathrm{O}_{2}$ at 0 h after serum shock; ${ }^{\$ \$ \$} P<0.0001$ vs. cells transfected with siRNA-Sc with $\mathrm{H}_{2} \mathrm{O}_{2}$ treatment at $0 \mathrm{~h}$ after serum shock; ${ }^{\wedge \wedge \wedge \wedge} P<0.0001$ vs. cells transfected with siRNA-Sc with $\mathrm{H}_{2} \mathrm{O}_{2}$ treatment at $12 \mathrm{~h}$ after serum shock; ${ }^{! ! !} P<0.0001$ vs. cells transfected with siRNA-Per 2 with $\mathrm{H}_{2} \mathrm{O}_{2}$ treatment at 0 h after serum shock). Alpha was set as 0.05 . Data are presented as the means \pm SEM. The Holm-Sidak method was used to correct for multiple $t$-tests.

were involved. The rate-limiting step for the entry of longchain fatty acids into the mitochondrial matrix is the synthesis of acylcarnitine from acyl CoA and carnitine, which is mediated by CPT1A [38]. It has been reported that the mRNA levels of Pdhb and Cpt1a also cycle for $24 \mathrm{~h}$ but reached their peak levels, respectively, at ZT16 and ZT17 [39]. Our results showed that the expression of Per2 and Cpt1a was basically similar, and peak expression of Per2 and Cpt1a coincided with the time when damage resistance of the heart was increased after the transition from light to dark, while that of $P d h b$ was different (Figure $4(\mathrm{~g})$ ). This indicated that PER2 damage to cardiomyocytes was mediated by CPT1A, which produced less ATP and provided insufficient energy to cardiomyocytes, thus leading to the aggravation of myocardial injury.

At present, the mechanisms linking circadian rhythm stabilization and myocardial infarction are under investigation and could involve inflammation [40], glycolysis metabolism [41], cyclin-dependent kinase 5 [42], or adenosine [33]. Some of these are known to be associated with PER2 and contribute to the survival of the myocardium after I/R. Sun et al. hypothesized that Per2 deficiency affected 
endothelial progenitor cell function through CXCR4/PI3$\mathrm{k} / \mathrm{Akt} / \mathrm{FoxO}$-related mechanisms involving angiogenesis in the ischemic myocardium of mice, thus increasing damage [43]. Kobayashi et al. established the dependence of HIF1A on PER2 as a transcription factor during hypoxia and proposed that PER2 acted as an effector molecule for the recruitment of HIF1A to promoter regions of its downstream genes [44]. On this basis, Oyama et al. also found that specific HIF1A pathways controlled glycolysis, mitochondrial respiration (COX4.2), or endothelial barrier function (ANGPTL-4/claudin-1) after PER2 overexpression [41].

Numerous studies have demonstrated links between myocardial infarction and disruption of circadian homeostasis both in clinical observations and in basic research findings. Therefore, it is important to maintain the homeostasis of circadian rhythms in humans. Interestingly, in addition to the above studies, chronic shift work [45], anesthetics [46], melatonin [47], and intestinal microbiota [48] can also affect circadian rhythms.

In summary, we have shown that the injury caused by myocardial infarctions has circadian rhythmicity. Our study suggests that PER2 deficiency contributed to the susceptibility of the myocardium to I/R injury, with peak susceptibility in the early morning, through reactive oxygen species and CPT1A as a key enzyme in energy metabolism, which may be particularly harmful to patients during a critical time window.

\section{Data Availability}

Data are available on request to the authors.

\section{Conflicts of Interest}

The authors declare that they have no known competing financial interests or personal relationships that influenced the work reported in this paper.

\section{Authors' Contributions}

Yaqian Weng, Hui Li, and Lin Gao contributed equally to this work. Shiyuan $\mathrm{Xu}$ and Le Li contributed equally to the article.

\section{Acknowledgments}

This research was supported by grants from the National Natural Science Foundation of China (Nos. 81873763 and 81771315).

\section{Supplementary Materials}

TABLE 1: primer sequences used in reverse transcriptionpolymerase chain reactions. TABLE 2: antibodies used for western blots. (Supplementary Materials)

\section{References}

[1] E. C. Keeley, J. A. Boura, and C. L. Grines, "Primary angioplasty versus intravenous thrombolytic therapy for acute myocardial infarction: a quantitative review of 23 randomised trials," The Lancet, vol. 361, no. 9351, pp. 13-20, 2003.
[2] E. Murphy and C. Steenbergen, "Mechanisms underlying acute protection from cardiac ischemia-reperfusion injury," Physiological Reviews, vol. 88, no. 2, pp. 581-609, 2008.

[3] E. T. Chouchani, V. R. Pell, A. M. James et al., “A Unifying Mechanism for Mitochondrial Superoxide Production during Ischemia- Reperfusion Injury," Cell Metabolism, vol. 23, no. 2, pp. 254-263, 2016.

[4] T. Kono, H. Morita, T. Nishina et al., "Circadian variations of onset of acute myocardial infarction and efficacy of thrombolytic therapy," Journal of the American College of Cardiology, vol. 27, no. 4, pp. 774-778, 1996.

[5] M. E. Young, P. Razeghi, A. M. Cedars, P. H. Guthrie, and H. Taegtmeyer, "Intrinsic diurnal variations in cardiac metabolism and contractile function," Circulation Research, vol. 89, no. 12, pp. 1199-1208, 2001.

[6] M. E. Young, P. Razeghi, and H. Taegtmeyer, "Clock genes in the heart: characterization and attenuation with hypertrophy," Circulation Research, vol. 88, no. 11, pp. 1142-1150, 2001.

[7] L. Li, H. Li, C. L. Tien, M. K. Jain, and L. Zhang, "Kruppel-like factor 15 regulates the circadian susceptibility to ischemia reperfusion injury in the heart," Circulation, vol. 141, no. 17, pp. 1427-1429, 2020.

[8] M. E. Young, "The circadian clock within the heart: potential influence on myocardial gene expression, metabolism, and function," American Journal of Physiology. Heart and Circulatory Physiology, vol. 290, no. 1, pp. H1-16, 2006.

[9] Y. Xia, Z. Cheng, S. Wang, D. Guan, and F. Liu, "Modeling the crosstalk between the circadian clock and ROS in _Neurospora crassa_," Journal of Theoretical Biology, vol. 458, pp. 125-132, 2018.

[10] F. C. Kelleher, A. Rao, and A. Maguire, "Circadian molecular clocks and cancer," Cancer Letters, vol. 342, no. 1, pp. 9-18, 2014.

[11] S. Bonney, K. Hughes, P. N. Harter, M. Mittelbronn, L. Walker, and T. Eckle, "Cardiac period 2 in myocardial ischemia: clinical implications of a light dependent protein," The International Journal of Biochemistry \& Cell Biology, vol. 45, no. 3, pp. 667-671, 2013.

[12] Y. Oyama, C. M. Bartman, J. Gile, D. Sehrt, and T. Eckle, “The circadian PER2 enhancer nobiletin reverses the deleterious effects of midazolam in myocardial ischemia and reperfusion injury," Current Pharmaceutical Design, vol. 24, no. 28, pp. 3376-3383, 2018.

[13] C. M. Bartman, Y. Oyama, K. Brodsky et al., "Intense lightelicited upregulation of miR-21 facilitates glycolysis and cardioprotection through Per2-dependent mechanisms," PLoS One, vol. 12, no. 4, article e0176243, 2017.

[14] M. C. Magnone, S. Langmesser, A. C. Bezdek, T. Tallone, S. Rusconi, and U. Albrecht, "The mammalian circadian clock gene per2 modulates cell death in response to oxidative stress," Frontiers in Neurology, vol. 5, 2015.

[15] P. A. K. Andersen, V. Petrenko, P. H. Rose et al., "Proinflammatory Cytokines Perturb Mouse and Human Pancreatic Islet Circadian Rhythmicity and Induce Uncoordinated $\beta$-Cell Clock Gene Expression via Nitric Oxide, Lysine Deacetylases, and Immunoproteasomal Activity," International Journal of Molecular Sciences, vol. 22, no. 1, p. 83, 2021.

[16] T. Eckle, M. Koeppen, and H. Eltzschig, "Use of a hanging weight system for coronary artery occlusion in mice," Journal of Visualized Experiments, vol. 50, 2011.

[17] T. Eckle, A. Grenz, D. Köhler et al., "Systematic evaluation of a novel model for cardiac ischemic preconditioning in mice," 
American Journal of Physiology. Heart and Circulatory Physiology, vol. 291, no. 5, pp. H2533-H2540, 2006.

[18] X. Liu, Y. Wang, P. Zhang, Q. Wang, Q. Feng, and W. Chen, "Radial motion estimation of myocardium in rats with myocardial infarction: a hybrid method of FNCCGLAM and polar transformation," Ultrasound in Medicine \& Biology, vol. 46, no. 12, pp. 3413-3425, 2020.

[19] K. Tanaka, N. Ashizawa, H. Kawano et al., "Aldosterone induces circadian gene expression of clock genes in H9c2 cardiomyoblasts," Heart and Vessels, vol. 22, no. 4, pp. 254-260, 2007.

[20] A. Balsalobre, F. Damiola, and U. Schibler, "A serum shock induces circadian gene expression in mammalian tissue culture cells," Cell, vol. 93, no. 6, pp. 929-937, 1998.

[21] J. X. Wang, X. J. Zhang, Q. Li et al., "MicroRNA-103/107 regulate programmed necrosis and myocardial ischemia/reperfusion injury through targeting FADD," Circulation Research, vol. 117, no. 4, pp. 352-363, 2015.

[22] W. Sun, L. Zhao, X. Song et al., "MicroRNA-210 modulates the cellular energy metabolism shift during $\mathrm{H}_{2} \mathrm{O}_{2}$-induced oxidative stress by repressing ISCU in $\mathrm{H} 9 \mathrm{c} 2$ cardiomyocytes," Cellular Physiology and Biochemistry, vol. 43, no. 1, pp. 383-394, 2017.

[23] N. Schallner, J. L. Lieberum, D. Gallo et al., "Carbon monoxide preserves circadian rhythm to reduce the severity of subarachnoid hemorrhage in mice," Stroke, vol. 48, no. 9, pp. 25652573, 2017.

[24] R. R. Almon, E. Yang, W. Lai, I. P. Androulakis, D. C. DuBois, and W. J. Jusko, "Circadian variations in rat liver gene expression: relationships to drug actions," The Journal of Pharmacology and Experimental Therapeutics, vol. 326, no. 3, pp. 700-716, 2008.

[25] T. Papagiannakopoulos, M. R. Bauer, S. M. Davidson et al., "Circadian rhythm disruption promotes lung tumorigenesis," Cell Metabolism, vol. 24, no. 2, pp. 324-331, 2016.

[26] N. Ohashi, S. Isobe, S. Ishigaki, and H. Yasuda, "Circadian rhythm of blood pressure and the renin-angiotensin system in the kidney," Hypertension Research, vol. 40, no. 5, pp. 413-422, 2017.

[27] N. Black, A. D’Souza, Y. Wang et al., "Circadian rhythm of cardiac electrophysiology, arrhythmogenesis, and the underlying mechanisms," Heart Rhythm, vol. 16, no. 2, pp. 298-307, 2019.

[28] H. O. Kim, J. M. Kim, J. S. Woo et al., "Circadian distribution of acute myocardial infarction in different age groups," The American Journal of Cardiology, vol. 121, no. 11, pp. 12791284, 2018.

[29] D. J. Durgan, T. Pulinilkunnil, C. Villegas-Montoya et al., "Short communication: ischemia/reperfusion tolerance is time-of-day-dependent: mediation by the cardiomyocyte circadian clock," Circulation Research, vol. 106, no. 3, pp. 546550, 2010.

[30] D. Rotter, D. B. Grinsfelder, V. Parra et al., "Calcineurin and its regulator, RCAN1, confer time-of-day changes in susceptibility of the heart to ischemia/reperfusion," Journal of Molecular and Cellular Cardiology, vol. 74, pp. 103-111, 2014.

[31] B. Grimaldi, M. M. Bellet, S. Katada et al., "PER2 Controls Lipid Metabolism by Direct Regulation of PPAR $\gamma$," Cell Metabolism, vol. 12, no. 5, pp. 509-520, 2010.

[32] S. H. Yoo, S. Kojima, K. Shimomura et al., "Period23'-UTR and microRNA-24 regulate circadian rhythms by repressing PERIOD2 protein accumulation," Proceedings of the National
Academy of Sciences of the United States of America, vol. 114, no. 42, pp. E8855-E8864, 2017.

[33] T. Eckle, K. Hartmann, S. Bonney et al., “Adora2b-elicited _Per2_ stabilization promotes a HIF-dependent metabolic switch crucial for myocardial adaptation to ischemia," Nature Medicine, vol. 18, no. 5, pp. 774-782, 2012.

[34] E. Fuentes, R. Moore-Carrasco, A. M. de Andrade Paes, and A. Trostchansky, "Role of platelet activation and oxidative stress in the evolution of myocardial infarction," Journal of Cardiovascular Pharmacology and Therapeutics, vol. 24, no. 6, pp. 509-520, 2019.

[35] W. Y. Wu, Y. D. Li, Y. K. Cui et al., “The natural flavone acacetin confers cardiomyocyte protection against hypoxia/reoxygenation injury via AMPK-mediated activation of Nrf2 signaling pathway," Frontiers in Pharmacology, vol. 9, p. 497, 2018.

[36] V. D. Ortiz, P. Türck, R. Teixeira et al., "Carvedilol and thyroid hormones co-administration mitigates oxidative stress and improves cardiac function after acute myocardial infarction," European Journal of Pharmacology, vol. 854, pp. 159-166, 2019.

[37] M. S. Patel, N. S. Nemeria, W. Furey, and F. Jordan, “The Pyruvate Dehydrogenase Complexes: Structure-based Function and Regulation*," The Journal of Biological Chemistry, vol. 289, no. 24, pp. 16615-16623, 2014.

[38] M. Schreurs, F. Kuipers, and F. R. van der Leij, "Regulatory enzymes of mitochondrial beta-oxidation as targets for treatment of the metabolic syndrome," Obesity Reviews, vol. 11, no. 5, pp. 380-388, 2010.

[39] A. Neufeld-Cohen, M. S. Robles, R. Aviram et al., "Circadian control of oscillations in mitochondrial rate-limiting enzymes and nutrient utilization by PERIOD proteins," Proceedings of the National Academy of Sciences of the United States of America, vol. 113, no. 12, pp. E1673-E1682, 2016.

[40] L. K. Fonken, T. A. Bedrosian, N. Zhang, Z. M. Weil, A. C. DeVries, and R. J. Nelson, "Dim light at night impairs recovery from global cerebral ischemia," Experimental Neurology, vol. 317, pp. 100-109, 2019.

[41] Y. Oyama, C. M. Bartman, S. Bonney et al., "Intense lightmediated circadian cardioprotection via transcriptional reprogramming of the endothelium," Cell Reports, vol. 28, no. 6, pp. 1471-1484.e11, 2019.

[42] A. Brenna, I. Olejniczak, R. Chavan et al., "Cyclin-dependent kinase 5 (CDK5) regulates the circadian clock," eLife, vol. 8, 2019.

[43] Y. Y. Sun, W. W. Bai, B. Wang et al., "Period 2 is essential to maintain early endothelial progenitor cell function in vitro and angiogenesis after myocardial infarction in mice," Journal of Cellular and Molecular Medicine, vol. 18, no. 5, pp. 907-918, 2014.

[44] M. Kobayashi, A. Morinibu, S. Koyasu, Y. Goto, M. Hiraoka, and H. Harada, "A circadian clock gene,PER2, activatesHIF1 as an effector molecule for recruitment ofHIF- $1 \alpha$ to promoter regions of its downstream genes," The FEBS Journal, vol. 284, no. 22, pp. 3804-3816, 2017.

[45] C. J. Morris, T. E. Purvis, J. Mistretta, K. Hu, and F. A. J. L. Scheer, "Circadian misalignment increases C-reactive protein and blood pressure in chronic shift workers," Journal of Biological Rhythms, vol. 32, no. 2, pp. 154-164, 2017.

[46] M. A. Wren-Dail, R. T. Dauchy, D. E. Blask et al., "Effect of isoflurane anesthesia on circadian metabolism and physiology in rats," Comparative Medicine, vol. 67, no. 2, pp. 138-146, 2017. 
[47] T. Y. Feng, Q. Li, F. Ren et al., "Melatonin protects goat spermatogonial stem cells against oxidative damage during cryopreservation by improving antioxidant capacity and inhibiting mitochondrial apoptosis pathway," Oxidative Medicine and Cellular Longevity, vol. 2020, Article ID 5954635, 16 pages, 2020.

[48] Z. Kuang, Y. Wang, Y. Li et al., "The intestinal microbiota programs diurnal rhythms in host metabolism through histone deacetylase 3," Science, vol. 365, no. 6460, pp. 1428-1434, 2019. 